\title{
FORMATION ET DIMENSION DE LA CULTURE DE GESTION DES FONCTIONNAIRES EN UKRAINE : VUE SOCIO-TECHNOLOGIQUE
}

\section{Volodymyr Perzhun}

Doctorat en Sciences Sociologiques, Professeur Assosié du Département de Philosophie et d'études Culturelles, Université Nationale au nom de Yuriy Fedkovych de Tchernivtsi;

Doctorant de l'Académie Nationale d'administration d'Etat, Ukraine e-mail: volodymyr_perzhun@ukr.net, orcid.org/0000-0001-8183-2888

\section{Andrzej Kryński}

Professor, Ph.D., Polonia University in Czestochowa, Interdisciplinary Faculty, Poland e-mail: a.krynski@ap.edu.pl, orcid.org/0000-0001-9635-023X

\section{Annotation}

La formation de la culture de gestion des fonctionnaires implique l'utilisation des technologies sociales qui, par leurs caractéristiques et leurs capacités, contribuent à l'efficacité de ce processus. Ils aident également à résoudre des problèmes difficiles, socioculturels, politiques, éthiques, etc. de gestion de l'activité. L'utilisation des technologies sociales dans le processus de formation de la culture de gestion des fonctionnaires est une sorte de moyen d'introduire la formation, l'éducation, les recherches sicenitifiques, les données empiriques, les connaissances, les compétences et les capacités pouvant être utilisées dans des activités pratiques de gestion. De plus, c'est une certaine manière de la pensée managériale, qui est utilisée pour identifier les sujets de la culture de gestion comme phénomène socio-politique de la société et de l'État, d'une part, et d'autre part - le processus d'établissement de la culture de gestion des fonctionnaires dans la pratique.

Dans le processus de développement et d'accumulation de la pensée managériale, une direction spéciale de la science de gestion moderne s'est formée, liée à la recherche, à la définition et à la sélection des régulateurs sociaux et managériaux de la formation de la culture de gestion. La tâche de ces régulateurs est de changer le contenu et les activités des objets sociaux, de former les connexions et les relations nécessaires entre eux.

La technologie est généralement inhérente aux activités humaines. Ils sont nés et établis avec l'homme en tant qu'être intelligent et la nature collective de l'activité. Tout d'abord, les technologies se sont concentrées sur la production de biens de consommation, puis les principes technologiques se sont étendus aux autres domaines de l'activité humaine: la production et l'application des connaissances, la reproduction de l'homme lui-même (un exemple de cette technologie est la formation et l'éducation). Aujourd'hui, une nouvelle tâche se pose - techniciser la reproduction de la société et de ses composants individuels.

Mots clés: société, technologies sociales, critères, valeurs, pensée de gestion, connaissances, expérience, compétences.

DOI: https://doi.org/10.23856/4526

\section{Introduction}

Au cours de son développement séculaire, l'humanité a accumulé un vaste arsenal de technologies diverses. Une place particulière parmi eux est occupée par les technologies sociales. La tâche principale de l'application des technologies sociales est, tout d'abord, la transformation 
de la société, les rapports et les relations sociales, politiques, économiques, culturelles, de gestions, les processus socio-politiques, la poursuite de la recherche et l'amélioration des régulateurs de diverses organisations, équipes, les communautés et les groupes, et finalement l'amélioration socioculturelle de l'homme lui-même. Cette formulation du problème est nouvelle, car elle est due au fait que la science de gestion, comme d'autres sciences, dans son développement aborde non seulement des questions scientifiques et théoriques et méthodologiques, mais aussi des applications pratiques, dans notre cas - la formation de la culture de gestion.

L’actualité de l'étude de la formation de la culture de gestion des fonctionnaires dans l'Ukraine moderne en termes d'approche socio-technologique ne doit pas être mise en doute, car il s'agit d'enquêtes sociologiques spécifiques sous forme d'expert et de questionnaire montrent les domaines problématiques les activités pratiques de gestion, qui sont actuellement les plus importantes dans le travail des fonctionnaires.

Le but de cette publication est d'analyser le concept de "technologie sociale", le rôle et l'importance des recherche empiriques dans la formation de la culture de gestion, afin de développer des recommandations pratiques pour les fonctionnaires. Ses principales missions sont: étudier les réalisations des scientifiques de premier plan qui ont été à l'origine du développement des technologies sociales, déterminer les critères inhérents aux technologies sociales, montrer la place et le rôle des régulateurs socio-technologiques dans la mesure de la culture de gestion, argumenter les données des recherches empiriques etc.

En analysant et en découvrant les problèmes actuels de formation de la culture de gestion des fonctionnaires en Ukraine, nous avons utilisé une enquête par questionnaire auprès des étudiants de l'Académie nationale d'administration publique du Président de l'Ukraine (ci-après ANAP) et de l'Institut de la formation permanente des employés de la formation, grâce aux efforts conjoints des scientifiques d'ANAP et de l'Université nationale au nom de Yuriy Fedkovych de Tchernivtsi dénommé (ci-après CHNU) selon le questionnaire «Manières de former la culture de gestion des fonctionnaires: approche empirique» (octobre - novembre 2019, 107 sondés ont été interrogés) et évaluation d'experts (16 étudiants du CHNU ont y participé) selon le questionnaire - Rôle et importance des relations interdisciplinaires des domaines de connaissance «Administration et gestion publique » (mars-avril 2021).

\section{L'essence, les critères, l'application des technologies sociales}

Le terme «technologie sociale» est apparu lorsqu'une personne a commencé à comprendre et à assimiler le milieu social, l'environnement social. Un rôle important dans l'introduction de la technologie dans la vie sociale a également joué une compréhension de la durabilité, le caractère cyclique des processus de reproduction de l'homme et de la société. En fait, l'application de l'approche socio-technologique, dans notre cas à la formation et à la mesure de la culture de gestion des fonctionnaires, est une manière spécialisée de mettre en œuvre les recherches scientifiques, les données empiriques, les connaissances, les compétences, les capacités et les idées, etc., qui peuvent être utilisées dans la gestion pratique. C'est une certaine manière de la pensée managériale, qui sert à identifier les sujets de la culture de gestion comme phénomène socio-politique de la société et de l'État, d'une part, et d'autre part - le processus de la culture des fonctionnaires dans la pratique.

Les origines de l'étude, la définition de l'essence et la mise en œuvre des technologies sociales dans la vie publique étaient des représentants bien connus de diverses branches de la science: philosophe G. Hegel, gestionnaire-praticien F. Taylor, sociologue et représentant de la pensée managériale $M$. Weber, représentant de la science de gestion et homme d'État 
W. Wilson, historien et sociologue M. Halbwax, politologues F. Paretto et G. Mosca, sociologue E. Giddens et autres. A l'étape actuelle, les technologies de gestion et la formation d'une culture de gestion acquièrent des signes, des caractéristiques et des essences quelque peu différentes. Notons en particulier: la théorie du changement institutionnel et son impact sur la gestion de D. North; renouvellement de l'État et du gouvernement sans bureaucrates D. Osborne et P. Plastrik; approches du nouveau management dans le domaine de la gestion des organisations G. Simon; caractéristiques socio-psychologiques de la gestion de B. Tracy; une nouvelle vision de la politique, de l'économie, de la culture et de la gestion dans le contexte de la mondialisation D. Held ; les nouvelles formes, principes et méthodes, y compris certaines technologies de gestion A. Heuser et autres.

L'application moderne des technologies sociales dans la formation de la culture de gestion des fonctionnaires, qui «représentent une nouvelle façon interdisciplinaire d'utiliser les connaissances et les idées existantes dans la réalité sociale pratique» (Sappa, 2009 : 51), comporte un ensemble de critères qui distinguent les activités technologiques par rapport aux autres. Ces critères sont inhérentes aux technologies matérielles et sociales. Citons les principaux:

1. La présence d'une activité transformationnelle, qui implique la transformation ellemême, pas seulement la connaissance de l'objet et, en outre, sépare la technologie de l'activité scientifique. Cette séparation de l'activité transformationnelle de l'activité cognitive sert d'orientation vers l'un ou l'autre objet. Dans le cas de l'activité scientifique et cognitive, son objet est toujours un système de connaissances, d'idées sur les mécanismes de fonctionnement de certains phénomènes (en particulier, la culture de gestion en tant que phénomène socio-politique et de gestion publique - auteur).

2. Définir l'objectif - la technologie agit comme un système d'action approprié. L'objectif est l'un des éléments du comportement humain et de l'activité consciente, qui se caractérise par l'anticipation dans la réflexion sur le résultat des activités et des moyens d'y parvenir par certains moyens (formation de la culture de gestion des fonctionnaires comme objectif; obtenir des résultats positifs dans ce processus comme un objectif - auteur).

3. Cohérence: l'activité se concentre non seulement sur l'objectif mais aussi sur les conditions externes, tout en ayant les propriétés d'auto-identification et d'organisation (parfois les processus de formation d'une culture de gestion peuvent être spontanés c'est l'influence des médias, des journaux et de la télévision aux réseaux sociaux, qui est difficile à réguler ainsi que les processus organisés de formation de la culture de gestion en tant qu'objet d'administration publique - auteur).

4. L'existence d'un ensemble de méthodes, car l'activité de transformation en technologie doit reposer sur l'utilisation de certaines méthodes disponibles pour la reproduction et celles qui permettent d'atteindre le résultat visé. Sur cette base, on peut affirmer que les technologies sociales sont «un ensemble systémique de méthodes pour changer l'état et les propriétés des objets sociaux ou changer la situation sociale pour atteindre un objectif prédéterminé» (Sappa, 2009 : 51).

Les critères ci-dessus, qui sont inhérentes aux technologies sociales et ont les propriétés de dimension sociale (pour nous, la culture de gestion des fonctionnaires) peuvent être utilisés dans la gestion pratique, la formation et la science de gestion, le recyclage des fonctionnaires du gouvernement central et du gouvernement local comme les domaines importants de la culture de gestion, qui sont introduits dans l'enseignement de diverses disciplines administratives, politiques, sociologiques, culturelles, philosophiques, historiques, etc. Par exemple, un régulateur ou un catalyseur efficace du processus de la formation de l'identité nationale et de la conscience nationale chez les fonctionnaires peut être un sentiment de dignité pour leur passé historique, 
le respect de leur peuple, la conscience de soi en tant qu'un ukrainien, des sentiments pour leur pays et position civile active. D'autres facteurs sont possibles - le désir de connaître les origines et la formation de la pensée managériale dans le monde et en Ukraine, l'amélioration des compétences de gestion dans la pratique, l'étude d'une expérience de gestion étrangère positive, l'utilisation des qualités de la culture de gestion pour une gestion efficace, l'application de nouvelles technologies, etc.

\section{Les régulateurs pour mesurer la culture de gestion}

Le régulateur socio-technologique le plus efficace pour mesurer la culture de gestion des fonctionnaires est une étude approfondie du cours de la pensée managériale, de la Grèce antique aux réalisations modernes des sciences de gestion. Des exemples sont : la doctrine de la «mesure en tout» du souverain d'Athènes et de l'homme d'État Solon; L'« art de gouverner » Socrate et Xénophon; «Cohérence dans la gestion» de Platon ; «Non-abus de pouvoir» d`Aristote et d'autres ; plus tard au Moyen Âge et au début de la Renaissance: forme politique et juridique - le «peuple législateur» du penseur italien M. Paduansky; attitude et verdict du peuple face aux managers du théoricien de la pensée politique et de gestion N. Machiavel; "Liberté de pensée" dans la gestion du philosophe hollandais B. Spinoza et d'autres. De plus, la doctrine de gestion de I. Kant - la gestion ne devrait pas être une coercition, parce que l'homme n'est pas un moyen pour un objectif, mais il est un tel objectif; l'opinion de G. Hegel selon laquelle la culture de la gestion est une activité de maintien de l'ordre, etc. De la fin du XIX ${ }^{\text {ème }}$ au début du XX ${ }^{\text {ème }}$ siècle: le concept de «facteur humain dans la gestion» N. Vitke ; principes du management scientifique de F. Taylor; la théorie de la gestion administrative d'A. Fayol; le concept de gestion ciblée de P. Drucker; la théorie de la bureaucratie idéale de M. Weber ; théorie des relations humaines E. Mayo et les autres. Enfin, les théories modernes du milieu du XX - début du XXI siècle : théorie des systèmes de la gestion de G. Simon; le concept d'influences de gestions d'A. Tannenbaum; principes de gestion efficace de T. Peters; le changement global et son impact sur la gestion de D. Held ; la dernière théorie de la gestion de G. Kuntz et d'autres. Aujourd'hui, les idées de gestion des scientifiques ukrainiens dans le domaine de la connaissance de la gestion de l'Ukraine indépendante, dont les principaux chercheurs travaillent avec succès: V. Bakumenko - les recherches théoriques et méthodologique sur les problèmes des décisions de gestion dans l'administration d'Etat; T. Vasylevska - normes éthiques du directeur de l'État; L. Gonyukova - le rôle et l'importance des partis et processus politiques dans l'administration publique; L. Pashko - la culture de gestion comme base des relations de gestions; O. Petroe - développement des questions d'évolution de la gestion de l'État à la gouvernance publique; M. Piren - le rôle de l'éducation dans la formation des professionnels de la gestion ; V. Perzhun - recherche des origines sociales et de gestions de la formation et de l'établissement de la culture de gestion, etc.

Ainsi, l'efficacité de la formation et de l'application ultérieure des critères de mesure de la culture de gestion des fonctionnaires est principalement liée au choix des régulateurs sociaux nécessaires pour atteindre l'objectif et le but, et la qualité de la conception des technologies d'impact nécessaires. Pour atteindre les buts et objectifs fixés, une approche socio-technologique du problème peut être utilisée, ce qui est nouveau manière interdisciplinaire d'utiliser les connaissances et les idées existantes dans la gestion pratique. C'est, en quelque sorte, une nouvelle façon de la pensée managériale, qui vise à rationaliser, changer ou former un système de relations publiques et étatiques, c'est-à-dire résoudre un problème pratique, identifier les régulateurs sociaux existants par rapport à une situation particulière et méthodes pour les faire 
fonctionner, qui peuvent être : l'intensité, l'efficacité, la clarté, la profondeur, la compréhension, l'autorité, etc. Par conséquent, la formation réussie de la culture de gestion des fonctionnaires est possible avec le soutien actif des institutions publiques et étatiques et le développement de régulateurs efficaces de ce processus et de critères pour mesurer le niveau de culture de gestion. Ces régulateurs, à notre avis, sont :

- les établissements d'enseignement supérieur, les organisations sociopolitiques, les sociétés à vocation nationale, les instituts de promotion de la fonction publique, les structures organisationnelles de l'administration et de la gestion publique, le pouvoir exécutif à différents niveaux, qui peuvent influencer la formation de la culture de gestion;

- le développement et la mise en œuvre d'un mécanisme normatif de régulation sociale et la formation d'un nouveau système d'administration publique ;

- rationalisation et la régulation des processus organisationnels et spontanés de formation de la culture de gestion des fonctionnaires;

- introduction de nouveaux cours et de cours spéciaux pour les étudiants. Ils peuvent être - l'histoire de la pensée managériale ; sociologie de l'administration publique; nouvelle philosophie de gestion; processus politiques modernes; avis de gestion en Ukraine; nouvelles théories de la gestion publique; culture de gestion : théorie et pratique ; les bases de la gestion dans les collectivités locales, etc.

\section{Le processus de formation d'une culture de gestion}

Pour étudier le processus de formation de la culture de gestion des fonctionnaires, l'auteur a mené deux études à l'aide d'un questionnaire. Les sondés au questionnaire étaient des étudiants de ANAP et de l'Institut de formation avancée des éducateurs (IFAE) (107 sondés, 2019). Les données de la recherche empirique nous permettent d'étudier et de faire une analyse précise de la formation de la culture de gestion des fonctionnaires en Ukraine.

L'étude « Manières de former la culture de gestion des fonctionnaires: une approche empirique» (Perzhun, Gonyukova, 2019) a identifié et a montré l'évaluation des sondés euxmêmes en termes de compréhension et d'importance pratique de la culture de gestion dans leur travail de gestion. À la question «Quelles qualités de la culture de gestion sont les plus importantes pour un fonctionnaire»? les sondés ont répondu: connaissance de l'industrie, hommes $-80,4$, femmes $-82,3 \%$; responsabilité, hommes $-74,7 \%$, femmes $-75,8 \%$; tolérance, hommes $-73,1 \%$, femmes $-77,8 \%$; transparence, hommes $-67,4 \%$, femmes $-65,8 \%$; tolérance et respect des citoyens, hommes - 57,8\%, femmes - 59,3\% (Perzhun, Gonyukova, 2019 : p. 17).

Ces données semblent optimistes. Les sondés comprennent et sont conscients de l'importance de la culture de gestion et identifient les principales qualités, à leur avis, qui devraient être les plus inhérentes aux fonctionnaires dans leur travail.

Dans une autre enquête (Rôle et importance des relations interdisciplinaires des domaines de connaissance «Administration et gestion publique». (Perzhun, 2021), qui a été menée en ligne (enquête Internet à distance, 16 sondés - étudiants de 3-5 années de CHNU) en 2021 année à la question: "La culture de la gestion des fonctionnaires fournit, à Votre avis, ce qui suit", on a donné les réponses suivantes: compréhension des changements structurels dans la société ukrainienne - 37,5\%; l'importance des principes scientifiques et institutionnels dans la formation de la culture de gestion $-31,3 \%$; déterminants socioculturels approbation de la culture de gestion - 37,5\%; défis de la mondialisation d'aujourd'hui - 31,3\%; coordination des travaux de toutes les branches du gouvernement $-37,5 \%$; relation efficace et efficiente 
entre le gouvernement central et le gouvernement local - 56, 3\%; connaissances, expérience, compétences pratiques, professionnalisme $-43,8 \%$; interaction politique et de gestion entre le gouvernement et les politiciens - 18, 8\% (Perzhun, 2021).

Bien entendu, ces données ne prétendent pas être complètes, car un petit nombre de sondés ont été interrogés et il s'agit actuellement d'étudiants qui se préparent à de futures activités, éventuellement dans le domaine de la gestion. Mais les tendances de leur compréhension de l'importance de la culture de gestion pour les fonctionnaires sont clairement visibles et c'est un fait positif.

\section{Critères de mesure des indicateurs du niveau de culture de gestion}

Pour un travail pratique plus efficace sur la formation de la culture de gestion, nous avons étudié, analysé et abordé de manière critique les questions scientifiques et pratiques importantes, nous avons développé des critères pour mesurer le niveau de culture de gestion. Il est également important de noter qu'elles ne sont pas définitives et ne prétendent en aucun cas à l'exhaustivité. C'est notre vision - ce qui peut être mesuré, quels sont les critères du niveau de culture de gestion des fonctionnaires. Ils peuvent et vont probablement changer, être complétés par d'autres caractéristiques, mais nous pensons que tels critères seront les plus utiles pour déterminer le niveau de culture de gestion d'un fonctionnaire public qui s'acquitte avec compétence ou pas tout à fait de ses fonctions. Ces critères et leurs manifestations comprennent: l'attitude envers leur travail (la gestion est une vocation, la responsabilité, la tolérance et l'indulgence, la formation, les connaissances et les compétences de l'industrie, la culture du travail et l'amélioration personnelle) ; indicateurs moraux et éthiques (comportement et actions de l'employé, valeurs sociales, sens de la distance entre le leader et le subordonné) ; caractéristiques juridiques (conscience des limites de gestions de l'activité, connaissance des évolutions structurelles de la gestion, respect des droits et des responsabilités, régulation interne et externe du comportement, formation d'une culture anti-corruption); normes organisationnelles et de gestions (ordre organisationnel, relations saines dans l'équipe, discipline interne, fixation de la charge de travail et exécution claire des tâches); cognition et pensée de gestions (approbation de la culture de gestion des fonctionnaires dans la pratique, amélioration des liens et des relations managériaux modernes, application des connaissances sur la pensée managériale).

Il est important, à notre avis, lorsque l'on considère les problèmes de technicisation de la formation de la culture de gestion, de prêter attention à l'opinion des fonctionnaires eux-mêmes sur leur niveau de culture de gestion.

Les sondés dans le cadre de l'étude «Manières de former la culture de gestion des fonctionnaires: une approche empirique» (Perzhun, Gonyukova, 2019) ont posé la question : «Comment décririez-vous le niveau de culture de gestion des fonctionnaires en général?». 53,4\% ont noté qu'au momemt actuel, les fonctionnaires sont au stade de la formation d'un nouveau type de culture de gestion. Dans le même temps, une partie importante des personnes interrogées $43,7 \%$ étaient d'avis que la culture de gestion des fonctionnaires est presque absente, et 16,9\% ont estimé que l'ancien type de culture de gestion est préservé (voir tableau 4.3.4.). Il est intéressant que les sondés qui ont répondu que l'ancien type de culture de gestion reste relativement plus ainé que leurs collègues (Perzhun, Gonyukova, 2019 : p. 19).

Le fait le plus préoccupant est que près de $45 \%$ des personnes interrogées ont clairement indiqué que la culture de la gestion est absente chez les fonctionnaires modernes. Ce fait alarme et confirme notre point de vue sur un certain manque de signes sains et de qualités de culture de gestion chez nos employés, managers, dirigeants, politiciens, etc. et l'urgence supplémentaire 
d'un travail efficace sur la formation de la culture de gestion des représentants des structures de gestion en Ukraine. Cette affirmation est confirmée par les réponses des agents publics à la question : «Quelles sont, à votre avis, les raisons d'une culture de gestion faible ou inexistante?». Les personnes interrogées mettent en premier lieu : les conséquences "post-soviétiques" et les vestiges de l'administration publique $-60,4 \%$. Parmi les raisons sont également citées : la réticence des fonctionnaires eux-mêmes à changer quelque chose, tandis que la situation actuelle dans le domaine de la culture de gestion leur convient» $-41,3 \%$; la complexité de la situation politique et administrative en Ukraine, qui affecte l'établissement d'une culture de gestion 39,7\%; faible niveau général de culture des fonctionnaires - 36,9\% ; possibilité insuffisante d'acquérir de nouvelles connaissances de la pensée managériale, niveau parfois faible de l'enseignement de la gestion et des autres disciplines $-14,5 \%$; il n'est pas toujours possible de suivre une reconversion professionnelle dans un établissement d'enseignement spécialisé, par exemple ANAP - 22,8\% (Perzhun, Gonyukova, 2019 : p. 20).

\section{Conclusions}

Les recherches empiriques et sociologiques nous permettent de développer quelques approches, conclusions et propositions socio-technologiques pour la formation de la culture de gestion des fonctionnaires. La première chose que je voudrais noter est que la culture de gestion n'est pas seulement l'accumulation de connaissances, mais aussi leur utilisation habile dans la pratique. La deuxième remarque importante est que les processus et les approches de formation de la culture de gestion des fonctionnaires montrent une tendance contradictoire : d'une part - il y a un besoin de formation pratique d'une culture de gestion mature, et d'autre part - l'utilisation non complète de potentiel dans sa formation, mais parfois comme le notent les sondés eux-mêmes les fonctionnaires ne sont pas toujours prêts pour d'éventuelles réformes tant dans la gestion que dans les changements d'approches pour l'établissement et l'adoption d'une nouvelle culture de gestion.

Dans le processus d'application des approches socio-technologiques à la formation de la culture de gestion des fonctionnaires, il est nécessaire d'utiliser plus efficacement les opportunités des établissements d'enseignement supérieur, des cours du recyclage des fonctionnaires d'Etat, des scientifiques traitant des questions d'administration publique, de politique, de gestion publique et de gestion des ressources humaines, des gestionnaires pratiques et des politiciens, organiser des conférences, des tables rondes, des discussions, des formations, etc. Cela sera facilité par l'utilisation de technologies sociales telles que : l'introduction de nouvelles méthodes d'enseignement de la gestion, des sciences politiques, de la sociologie, de la philosophie, de la culture, qui révéleront aux étudiants leurs connaissances en gestion et les caractéristiques et les qualités de la culture de gestion; compréhension et attitude envers les réformes modernes; participation éventuelle des fonctionnaires aux réformes administratives ; développement par les enseignants de nouveaux cours spéciaux sur la gestion d’Etat (publique): «La culture de la gestion en Ukraine: histoire et modernité», «Dernières réalisations de la pensée mondiale de la gestion», «Nouvelle gestion publique: problèmes de formation et de préservation», «Gestion des ressources humaines», «Gestion de projet», "Gestion stratégique» et d'autres visant à former la culture de gestion des fonctionnaires ; augmenter les exigences en matière d'éducation et de culture individuelles en gestion, en droit, en politique, en culture d'un fonctionnaire public, etc.

Le processus de formation de la cognition et de la pensée socio-politique et de gestion, sur la base duquel les besoins et les compétences de reconstitution de son bagage théorique, 
sa compréhension critique et sa corrélation avec la dynamique des processus sociaux, économiques, politiques, culturels, managériaux, est insuffisant pour les fonctionnaires, se produisent aujourd'hui dans la société et dans l'État. Les connaissances, l'expérience sociale et managériale et les compétences acquises par de nombreux fonctionnaires ne trouvent pas toujours leur application dans la pratique. Ainsi, pour fournir des conditions appropriées au travail des scientifiques dans le domaine de l'administration publique, des gestionnaires, des fonctionnaires, leur participation à l'élaboration de recommandations et propositions spécifiques sur les plans économique, social, politique, culturel, managérial est urgent. En d'autres termes, les fonctionnaires, en particulier les jeunes, ont besoin de voir la perspective, et s'il y en a, ils pourront se réaliser pleinement.

De manière générale, en résumant les problèmes d'approches socio-technologiques de la formation et de la mesure des critères de la culture de gestion des fonctionnaires, il convient de noter que la modernité avec ses situations conflictuelles et ses contradictions nécessite de trouver et d'approuver de nouvelles technologies et méthodes sociales, des justifications théoriques et des étapes pratiques pour la gestion, les activités, la formation, l'éducation et la formation d'une nouvelle génération de fonctionnaires de l'Ukraine moderne. Le problème reste ouvert et nécessite une enquête plus approfondie. Il existe des opportunités potentielles pour cela, car la vie ne s'arrête pas, elle change et, avec elle, change les technologies sociales, les approches, les méthodes, les principes, les principes spirituels et moraux de formation et d'approbation de la culture de gestion des fonctionnaires en Ukraine.

\section{Littérature}

Perzhun V. V. (2021). Rôle et importance des relations interdisciplinaires des domaines de connaissance "Administration et gestion publique». Enquête sociologique d'experts. Tchernivtsi : CHNU. [Ressource électronique]. Mode d'accès : https://drive.google.com/drive/folders/1mMG7fgxViGBxYP-gJ3a9xO-JJMkgpNxN? usp=sharing

Perzhun V. V., Gonyukova L. V. (2019). Manières de former la culture de gestion des fonctionnaires : une approche empirique. Tchernivtsi : Ruta.

Sappa M. M. (2009). Les technologies sociales dans la structure des connaissances sociologiques. Politique de jeunesse : problèmes et perspectives : Collection de résumés Conférence internationale scientifique-pratique. Drohobych : Maison d'édition DDPU. 